\title{
ON THE STARK BROADENING OF Ar VII SPECTRAL LINES
}

\author{
MILAN S. DIMITRIJEVIĆ ${ }^{*}$, SYLVIE SAHAL-BRÉCHOT ${ }^{2}$ \\ ${ }^{1}$ Astronomical Observatory, Volgina 7, Belgrade, Serbia \\ ${ }^{2}$ LERMA, Observatoire de Paris, PSL Research University, CNRS, Sorbonne Universities, UPMC Univ. Paris 06, 5 Place \\ Jules Janssen, Meudon Cedex, France
}

\begin{abstract}
Stark broadening parameters, full width at half maximum of s pectral line and shift, have been calculated for 3 spectral lines of Ar VII, for broadening by electron, proton, and He III impacts. For calculations, the semiclassical perturbation approach in the impact approximation has been used. The results are provided for temperatures from $20000 \mathrm{~K}$ to $500000 \mathrm{~K}$ and for an electron density of $10^{17} \mathrm{~cm}^{-3}$. Obtained results will be included in the STARK-B database which is also included in Virtual atomic and molecular data center (VAMDC).
\end{abstract}

Keywords: Stark broadening, atomic data, atomic processes, line profiles.

\section{INTRODUCTION}

Data on the Stark broadening of spectral lines are important for diagnostics, modeling and investigation of laboratory plasmas (Konjević, 1999; Torres et al., 2006), inertial fusion plasma research (Griem, 1992), laser produced plasma analysis and diagnostics (Gornushkin et al., 1999; Sorge et al., 2000), as well as for different plasmas in technology, like for example for welding and piercing with laser produced plasma (Hoffman et al., 2006), or for design and development of light sources based on plasmas (Dimitrijević \& Sahal-Bréchot, 2014a), and lasers (Csillag \& Dimitrijević, 2004). An important topic where Stark broadening data are needed are astrophysical plasmas (Beauchamp et al., 1997; Dimitrijević \& Sahal-Bréchot, 2014b), in particular atmospheres of white dwarfs, pre white dwarf stars, and post AGB (Asymptotic Giant Branch) stars (Tankosić et al., 2003; Milovanović et al., 2004; Simić et al., 2006; Dufour et al., 2011).

Additionally, since for temperatures higher than around 10 $000 \mathrm{~K}$ hydrogen is mainly ionized, Stark broadening is, in such a case, the principal pressure broadening mechanism (Griem, 1974). Consequently, in some atmospheric layers of A and late B stars, where such plasma conditions are typical, it should be taken into account. Such cases have been analysed for example in Simić et al. (2005b,a) and Simić et al. (2009).

Due to development of satellite-born spectros copy, data on trace elements, which have been without significance for astrophysics, become more and more important for analysis of stellar spectra. It is also worth to notice that Rauch et al. (2007) accentuated that accurate Stark broadening data for as much as possible large number of atoms and ions and the corresponding spectral lines "are of crucial importance for sophisticated analysis of stellar spectra by means of NLTE model atmospheres".
Spectral lines of Ar VII have been found by Taresch et al. (1997) in the spectrum of extremely hot and massive galactic O3 If supergiant HD 93129A. Also Werner et al. (2007), by analyzing high-resolution spectra taken with the Far Ultraviolet Spectroscopic Explorer (FUSE), have identified Ar VII lines in some of the hotest known central stars of planetary nebulae, with the effective temperatures of $95000-110000 \mathrm{~K}$, and in (pre-) white dwarfs, where Stark broadening is very important and the corresponding Stark brooadening data are needed for a reliable analysis and modelling.

In order to provide the needed Stark broadening parameters for Ar VII spectral lines, completely missing in the existing literature, Stark Full Widths at Half intensity Maximum (FWHM) $W$ and shifts $d$ for three transitions have been calculated by using semiclassical perturbation method (SCP, Sahal-Bréchot $(1969 \mathrm{a}, \mathrm{b}))$ for colissions of Ar VII ions with electrons, protons and He III ions, which are the main constituents of stellar at mospheres.

\section{THE IMPACT S EMICLASSICAL PERTURB ATION METHOD}

For the calculations of Stark broadening parameters, full width at half intensity maximum (FWHM - W) and shift of spectral line $(d)$ here is usede the semiclassical perturbation formalis m (SCP), developed in Sahal-Bréchot (1969a,b). Further innovations and modernisations are presented in Sahal-Bréchot (1974, 1991); Dimitrijević et al. (1991); Dimitrijević \& SahalBréchot (1996); Sahal-Bréchot et al. (2014).

The Stark broadened profile $F(\omega)$ of an isolated spectral line has Lorentzian form and can be represented as:

$$
F(\omega)=\frac{W / 2 \pi}{\left(\omega-\omega_{i f}-d\right)^{2}+(W / 2)^{2}} .
$$

Here,

\footnotetext{
* Corresponding author: mdimitrijevic@aob.rs
} 


$$
\omega_{i f}=\frac{E_{i}-E_{f}}{\hbar} .
$$

In the upper equation $E_{i}$ and $E_{f}$ are energies of the initial and final states, while $(W)$ and $(d)$, in angular frequency units, are given by equation:

$$
\begin{gathered}
W=N \int v f(v) d v\left(\sum_{i^{\prime} \neq i} \sigma_{i i^{\prime}}(v)+\sum_{f^{\prime} \neq f} \sigma_{f f^{\prime}}(v)+\sigma_{e l}\right), \\
d=N \int v f(v) d v \int_{R_{3}}^{R_{D}} 2 \pi \rho d \rho \sin \left(2 \varphi_{p}\right) .
\end{gathered}
$$

with $N$ is here denoted the electron density and with $\mathrm{f}(v)$ the Maxwellian velocity distribution function for electrons, $\rho$ is the impact parameter of the incoming electron, and with $i^{\prime}, f^{\prime}$ are denoted the perturbing levels of the initial and final state. The inelastic cross section $\sigma_{j j}$, $(v), j=i, f$ is given with the formula:

$$
\sum_{i^{\prime}=i} \sigma_{i i^{\prime}}(v)=\frac{1}{2} \pi R_{1}^{2}+\int_{R_{1}}^{R_{D}} 2 \pi \rho d \rho \sum_{i^{\prime}=i} P_{i i^{\prime}}(\rho, v) .
$$

where $P_{J j}(\rho, v), j=i, f, j^{\prime}=i^{\prime}, f^{\prime}$ is transition probability. The elastic cross section is

$$
\begin{gathered}
\sigma_{e l}=2 \pi R_{2}^{2}+\int_{R_{1}}^{R_{D}} 2 \pi \rho d \rho \sin ^{2} \delta+\sigma_{r}, \\
\delta=\left(\varphi_{p}^{2}+\varphi_{q}^{2}\right)^{\frac{1}{2}} .
\end{gathered}
$$

The phase shifts due to the polarization potential is $\varphi_{p}\left(r^{-4}\right)$ and due to the quadrupolar potential $\varphi_{q}\left(r^{-3}\right)$. They are defined in Section 3 of Chapter 2 in Sahal-Bréchot (1969a). The cut-offs $R_{1}$, $R_{2}, R_{3}$, and the Debye radius $R_{D}$ are described in Section 1 of Chapter 3 in Sahal-Bréchot (1969b). The contribution of Feshbach resonances, $\sigma_{r}$ is explained in details in (Fleurier et al., 1977).

All approximations and the details of the theory are discussed in detail in Sahal-Bréchot et al. (2014). The electrons are moving along hyperbolic paths while for ionic perturbers the paths are different because for them the Coulomb force is repulsive and for them, in Eqs. (2-4), there is no the contribution of Feshbach resonances.

\section{STARK B ROADENING PARAMETER CALCULATIONS}

Within the frame of semiclassical perturbation theory (Sahal-Bréchot, 1969a,b; Sahal-Bréchot et al., 2014) we have calculated using Eqs. (2-4) widths (FWHM) and shifts for three multiplets of six time charged argon ion Ar VII. Energy levels necessary for present calculations have been taken from Salo man (2010). The needed oscillator strengths have been calculated within the Coulomb approximation by using the method of Bates \& Damgaard (1949) and the tables of Oertel \& Shomo (1968). For higher levels, when there is no the corresponding data in Oertel \& Shomo (1968), the required oscillator strengths have been calculated according to the article of Van Regemorter et al. (1979).
In Table 1, the obtained results of our calculations of Stark widths (FWHM) and shifts for electron- proton- and doubly charged helium ion-impact broadening, for a perturber density of $10^{17} \mathrm{~cm}^{-3}$ and for a set of temperatures from $20000 \mathrm{~K}$ to 500000 $\mathrm{K}$, are shown. The temperature range covers needs in astrophysics, laboratory plasma, fusion research, technology and the topic of lasers and laser produced plasma. Extrapolation to perturber densities lower than $10^{17} \mathrm{~cm}^{-3}$ is linear. For higher perturber densities the influence of Debye screening should be checked and eventualy taken into account (see e.g. (Griem, 1974)). With known Stark broadening parameters, $W$ and $d$ it is easy to obtain the line profile using Eq. (1).

One can see from Table 1, that spectral line width due to collisions with electrons is always dominant in comparis on with line widths produced by ionic collisions, since ions are much heavier than electrons and their velocities are much smaller. At low temperatures ion width is completely neglibigle but its influence increases with temperature and it should not be neglected at high temperatures. One can see as well that widths due to collisions with doubly charged helium ions are larger than widths produced by collisions with protons. Shifts are much smaller and they are of the same order of magnitude for collisions with electrons as well as with both species of ions. At higher temperatures ion shifts are larger than electron ones and proton shifts are smaller than $\mathrm{He}^{++}$ones.

It should be noticed that wavelengths given in Table 1 are calculated ones, so that they are different from experimental ones. However, they are correct in angular frequency units since then, for the calculation of Stark broadening parameters, relative and not absolute positions of energy levels are significant. In order to transform the Stark widths in $\AA$-units to the width in angular frequency units the following formula can be used:

$$
W(\AA)=\frac{\lambda^{2}}{2 \pi c} w\left(s^{-1}\right) .
$$

where $c$ is the speed of light. If the correction of widths and/or shifts for the difference between calculated and experimental wavelength is needed, this can be performed for the width as:

$$
W_{\text {cor }}=\left(\frac{\lambda_{\text {exp }}}{\lambda}\right)^{2} W .
$$

Here, with $W_{\text {cor }}$ is denoted the corrected width, $\lambda_{\exp }$ is the experimental, $\lambda$ the calculated wavelength and $W$ the width from Table 1. Formulas for the shifts are analogous to Eqs. (5) and (6).

Parameter $C$ (Dimitrijević \& Sahal-Bréchot, 1984) in Table 1 , enables to estimate the maximal perturber density for which the line may be treated as isolated, when it is divided by the corresponding full width at half maximu m.

\section{ON THE IMPLEMENTATION OF RESULTS IN THE STARK-B DATAB ASE}

The presented in Table 1 Stark broadening parameters for Ar VII spectral lines, will be also implemented in the STARK-B 
database (Sahal-Bréchot et al., 2015, 2017), intended for the investigations, modelling and diagnostics of the plasma of stellar atmospheres, diagnostics of laboratory plasmas, and investigation of laser produced, inertial fusion plasma and for plasma technologies.

The STARK-B database contains Stark widths and shifts calculated by authors of this article and their coauthors, by using the SCP computer code for spectral lines, and published in more than 150 publications. Actually, in this database are SCP data for the following elements and ionization degrees: Ag I, Al I, Al III, Al XI, Ar I, Ar II, Ar III, Ar VIII, Au I, B II, B III, Ba I, Ba II,
Be I, Be II, Be III, Br I, C II, C III, C IV, C V, Ca I, Ca II, Ca V, Ca IX, Ca X, Cd I, Cd II, Cl I, Cl VII, Cr I, Cr II, Cu I, F I, F II, F III, F IV, F V, F VI, F VII, Fe II, Ga I, Ge I, Ge IV, He I, Hg II, I I, In II, In III, K I, K VIII, K IX, Kr I, Kr II, Kr VIII, Li I, Li II, Mg I, Mg II, Mg XI, Mn II, N I, N II, N III, N IV, N V, Na I, $\mathrm{Na} X, \mathrm{Ne} I, \mathrm{Ne}$ II, Ne III, Ne IV, Ne V, Ne VIII, Ni II, O I, O III, O IV, O V, O VI, O VII, P IV, P V, Pb IV, Pd I, Rb I, S III, S IV, S V, S VI, Sc III, Sc X, Sc XI, Se I, Si I, Si II, Si IV, Si V, Si VI, Si XI, Si XII, Si XIII, Sr I, Te I, Ti IV, Ti XII, Ti XIII, T1 III, V V, V XIII, Y III, Xe VI, Xe VIII and Zn I.

Table 1. This table gives electron-, proton-, and doubly charged helium-impact broadening parameters for Ar VII spectral lines, for a perturber density of $10^{17} \mathrm{~cm}^{-3}$ and temperatures from 20000 to $500000 \mathrm{~K}$. Calculated wavelength of the transitions (in $\AA$ ) and parameter $C$ are also given. This parameter, when divided with the corresponding Stark width, gives an estimate for the maximal pertuber density for which the line may be treated as isolated. $\mathrm{W}_{e}$ : electron-impact full width at half maximum of intensity, $d_{e}$ : electron-impact shift, $\mathrm{W}_{p}$ : proton-impact full width at half maximum of intensity, $d_{p}$ : proton-impact shift, $\mathrm{W}_{H e++}$ : doubly charged helium ion-impact full width at half maximu m of intensity, $d_{\mathrm{He+}}$ : doubly charged helium ion-impact shift.

\begin{tabular}{|c|c|c|c|c|c|c|c|}
\hline Transition & $\mathrm{T}(\mathrm{K})$ & $\mathrm{W}_{e}(\AA)$ & $d_{e}(\AA)$ & $\mathrm{W}_{H+}(\AA)$ & $d_{H+}(\AA)$ & $\mathrm{W}_{\mathrm{He++}}(\AA)$ & $d_{H e++}(\AA)$ \\
\hline Ar VII $3 s^{2}{ }^{1} S-3 p{ }^{1 P o}$ & 20000. & $0.144 \mathrm{E}-02$ & $0.504 \mathrm{E}-04$ & $0.414 \mathrm{E}-06$ & $-0.397 \mathrm{E}-06$ & $0.789 \mathrm{E}-06$ & $-0.716 \mathrm{E}-06$ \\
\hline $585.7 \AA$ & 50000. & $0.919 \mathrm{E}-03$ & $-0.130 \mathrm{E}-05$ & $0.164 \mathrm{E}-05$ & $-0.109 \mathrm{E}-05$ & $0.316 \mathrm{E}-05$ & $-0.210 \mathrm{E}-05$ \\
\hline \multirow[t]{4}{*}{$C=0.59 E+20$} & 100000. & $0.653 \mathrm{E}-03$ & $-0.449 \mathrm{E}-05$ & $0.447 \mathrm{E}-05$ & $-0.221 \mathrm{E}-05$ & $0.865 \mathrm{E}-05$ & $-0.438 \mathrm{E}-05$ \\
\hline & 200000 . & $0.465 \mathrm{E}-03$ & $-0.587 \mathrm{E}-05$ & $0.106 \mathrm{E}-04$ & $-0.434 \mathrm{E}-05$ & $0.206 \mathrm{E}-04$ & $-0.869 \mathrm{E}-05$ \\
\hline & 300000. & $0.386 \mathrm{E}-03$ & $-0.496 \mathrm{E}-05$ & $0.155 \mathrm{E}-04$ & $-0.621 \mathrm{E}-05$ & $0.304 \mathrm{E}-04$ & $-0.124 \mathrm{E}-04$ \\
\hline & 500000. & $0.309 \mathrm{E}-03$ & $-0.637 \mathrm{E}-05$ & $0.222 \mathrm{E}-04$ & $-0.902 \mathrm{E}-05$ & $0.438 \mathrm{E}-04$ & $-0.181 \mathrm{E}-04$ \\
\hline Ar VII $4 s^{1} S-4 p{ }^{1 P o}$ & 20000. & $0.860 \mathrm{E}-01$ & $0.166 \mathrm{E}-03$ & $0.126 \mathrm{E}-03$ & $-0.194 \mathrm{E}-03$ & $0.241 \mathrm{E}-03$ & $-0.349 \mathrm{E}-03$ \\
\hline $2445.8 \AA$ & 50000. & $0.546 \mathrm{E}-01$ & $-0.106 \mathrm{E}-02$ & $0.488 \mathrm{E}-03$ & $-0.524 \mathrm{E}-03$ & $0.948 \mathrm{E}-03$ & $-0.101 \mathrm{E}-02$ \\
\hline \multirow[t]{4}{*}{$\mathrm{C}=0.24 \mathrm{E}+21$} & 100000. & $0.396 \mathrm{E}-01$ & $-0.903 \mathrm{E}-02$ & $0.109 \mathrm{E}-02$ & $-0.952 \mathrm{E}-03$ & $0.215 \mathrm{E}-02$ & $-0.189 \mathrm{E}-02$ \\
\hline & 200000. & $0.297 \mathrm{E}-01$ & $-0.126 \mathrm{E}-02$ & $0.186 \mathrm{E}-02$ & $-0.145 \mathrm{E}-02$ & $0.369 \mathrm{E}-02$ & $-0.292 \mathrm{E}-02$ \\
\hline & 300000. & $0.255 \mathrm{E}-01$ & $-0.121 \mathrm{E}-02$ & $0.239 \mathrm{E}-02$ & $-0.176 \mathrm{E}-02$ & $0.474 \mathrm{E}-02$ & $-0.355 \mathrm{E}-02$ \\
\hline & 500000. & $0.213 \mathrm{E}-01$ & $-0.113 \mathrm{E}-02$ & $0.289 \mathrm{E}-02$ & $-0.210 \mathrm{E}-02$ & $0.578 \mathrm{E}-02$ & $-0.425 \mathrm{E}-02$ \\
\hline Ar VII $3 s^{3} S-4 p{ }^{3 P o}$ & 20000. & $0.534 \mathrm{E}-01$ & $0.172 \mathrm{E}-02$ & $0.781 \mathrm{E}-04$ & $-0.877 \mathrm{E}-04$ & $0.149 \mathrm{E}-03$ & $-0.158 \mathrm{E}-03$ \\
\hline $1982.0 \AA$ & 50000. & $0.333 \mathrm{E}-01$ & $-0.387 \mathrm{E}-03$ & $0.295 \mathrm{E}-03$ & $-0.239 \mathrm{E}-03$ & $0.571 \mathrm{E}-03$ & $-0.460 \mathrm{E}-03$ \\
\hline \multirow[t]{4}{*}{$C=0.20 E+21$} & 100000. & $0.240 \mathrm{E}-01$ & $-0.512 \mathrm{E}-03$ & $0.653 \mathrm{E}-03$ & $-0.449 \mathrm{E}-03$ & $0.128 \mathrm{E}-02$ & $-0.892 \mathrm{E}-03$ \\
\hline & 200000. & $0.179 \mathrm{E}-01$ & $-0.607 \mathrm{E}-03$ & $0.110 \mathrm{E}-02$ & $-0.714 \mathrm{E}-03$ & $0.219 \mathrm{E}-02$ & $-0.144 \mathrm{E}-02$ \\
\hline & 300000. & $0.153 \mathrm{E}-01$ & $-0.649 \mathrm{E}-03$ & $0.139 \mathrm{E}-02$ & $-0.865 \mathrm{E}-03$ & $0.277 \mathrm{E}-02$ & $-0.174 \mathrm{E}-02$ \\
\hline & 500000. & $0.128 \mathrm{E}-01$ & $-0.572 \mathrm{E}-03$ & $0.169 \mathrm{E}-02$ & $-0.107 \mathrm{E}-02$ & $0.338 \mathrm{E}-02$ & $-0.216 \mathrm{E}-02$ \\
\hline
\end{tabular}

STARK-B contains also our Stark broadening data obtained by using the Modified SemiEmpirical method (MSE) (Dimitrijević \& Konjević, 1980; Dimitrijević \& Kršljanin, 1986; Dimitrijević \& Popović, 2001).These data are of lower accuracy than SCP data but this approach is convenient for the cases where atomic data are not sufficiently complete for an adequate SCP calculation. MSE Stark line widths, in some cases together with line shifts, of the following emitters are in STARK-B 
database: Ag II, Al III, Al V, Ar II, Ar III, Ar IV, As II, Au II, B III, B IV, Be III, Bi II, Bi III, Br II, C III, C IV, C V, Cd II, Cd III, ClIII, ClIV, Cl VI, Co II, Co III, Cu III, Cu IV, Eu II, Eu III, F III, F V, F VI, Fe II, Ga III, Ge III, Ge IV, I II, Kr II, Kr III, La II, La III, Lu III, Mg III, Mg IV, Mn II, Mn III, N II, N III, Na III, Na VI, Nb III, Ne III, Ne IV, Ne V, Ne VI, Ne VII, Ne VIII, O III, O IV, P III, P IV, P VI, Pt II, Ra II, S IV, Sb II, Sc II, Se II, Se III, Si IV, Si V, Si VI, Si XI, Sn III, Sr III, Ti II, Ti III, V II, V III, V IV, Xe II, Y II, Zn II, Zn III, Zr II and Zr III.

We note as well that STARK-B database is one of the databases which are in the Virtual Atomic and Molecular Data Center - VAMDC (Dubernet et al., 2010; Rixon et al., 2011; Dubernet et al., 2016). VAMDC is created in order to enable an efficacious search and mining of atomic and molecular data scattered in different databases and to make more convenient their adequate use. It has been the principal aim of a FP7 founded project of the same name (Dubernet et al., 2010), which started on July 12009 and lasted 42 months. During this project an interoperable e-infrastructure for atomic and molecular data upgrading and integrating European (and wider) A\&M database services has been build, and a forum of data producers, data users and databases developers has been created. Currently in VAMDC are 30 databases with atomic and molecular data, including STARK-B, and can be accessed and searched through VAMDC portal: http://portal.vamdc.org/. The web site of VAMDC Consortium is: http://www.vamdc.org/.

\section{CONCLUSION}

We have performed a SCP calculation of Stark broadening parameters for three multiplets of Ar VII. Stark broadening parameters - widths and shifts, have been calculated for collisions of Ar VII ions with electrons, protons and doubly charged helium ions. The obtained data will be implemented in STARK-B database. There is no neither theoretical, nor experimental data for Stark broadening of Ar VII spectral lines and we hope that the obtained results will be of interest. for a number of problems in astrophysical, laboratory, laser produced, inertial fusion and technological plasmas.

\section{ACKNOWLEGMENTS}

This work is a part of the project 176002 "Influence of collisional processes on astrophysical plasma line shapes" supported by the Ministry of Education, Science and Technological Develop ment of Serbia.

\section{REFERENCES}

Bates, D.R., \& Damgaard, A. 1949. The Calculation of the Absolute Strengths of Spectral Lines. Philosophical Transactions of the Royal Society of London. Series A. Mathematical and Physical Sciences, 242(842), pp. 101-122.
Beauchamp, A., Wesemael, F., \& Bergeron, P. 1997. Spectroscopic Studies of DB White Dwarfs: Improved Stark Profiles for Optical Transitions of Neutral Helium. Astrophysical Journal Supplement, 108, pp. 559573.

Dimitrijevic, M.S., \& Csillag, L. 2004. On the Stark broadening of the $537.8 \mathrm{~nm}$ and $441.6 \mathrm{~nm} \mathrm{Cd}+$ lines excited in a hollow cathode laser discharge. Applied Physics B: Lasers and Optics, 78(2), pp. 221-223. doi:10.1007/s00340-003-1368-3

Dimitrijević, M.S., \& Konjević, N. 1980. Stark widths of doublyand triply-ionized atom lines. Journal of Quantitative Spectroscopy \& Radiative Transfer, 24, pp. 451-459.

Dimitrijević, M.S., \& Kršljanin, V. 1986. Electron-impact shifts of ion lines: Modified semiempirical approach. Astronomy and Astrophysics, 165, pp. 269-274.

Dimitrijević, M.S., \& Popović, L.Č. 2001. Modified Semiempirical Method,. Journal of Applied Spectroscopy, 68(6), pp. 893-901. doi:10.1023/A:1014396826047

Dimitrijević, M.S., \& Sahal-Bréchot, S. 1984. Stark broadening of neutral helium lines. Journal of Quantitative Spectroscopy \& Radiative Transfer, 31, pp. 301-313.

Dimitrijević, M.S., \& Sahal-Bréchot, S. 1996. Stark broadening of Li II spectral lines. Physica Scripta, 54, pp. 50-55.

Dimitrijević, M.S., \& Sahal-Bréchot, S. 2014. On the Application of Stark Broadening Data Determined with a Semic lassical Perturbation Approach. Atoms, 2, pp. 357-377.

Dimitrijević, M.S., Sahal-Bréchot, S., \& Bommier, V. 1991. Stark broadening of spectral lines of multicharged ions of astrophysical interest. I - C IV lines. Astronomy and Astrophysics Supplement Series, 89, pp. 581-590.

Dubernet, M.L., Antony, B.K., Ba, Y.A., \& et al., 2016. The virtual atomic and molecular data centre (VAMDC) consortium. Journal of Physics B: Atomic, Molecular and Optical Physics, 49(7). 074003.

Dubernet, M.L., Boudon, V., Culhane, J.L., \& et al., 2010. Virtual atomic and molecular data centre. Journal of Quantitative Spectroscopy \& Radiative Transfer, 111(15), pp. 2151-2159.

Dufour, P., Nessib, B.N., Sahal-Bréchot, S., \& Dimitrijević, M.S. 2011. Stark Broadening of Carbon and Oxygen Lines in Hot DQ White Dwarf Stars: Recent Results and Applications. Balt ic Astronomy, 20, pp. 511-515.

Fleurier, C., Sahal-Bréchot, S., \& Chapelle, J. 1977. Stark profiles of some ion lines of alkaline earth elements. Journal of Quantitative Spectroscopy and Radiative Transfer, 17, pp. 595-603.

Go rnushkin, I.B., King, L.A., Smith, B.W., Omenetto, N., \& Winefordner, J.D. 1999. Line broadening mechanisms in the low pressure laser-induced plasma. Spectrochimica Acta, Part B: Atomic Spectroscopy, 54(8), pp. 1207-1217.

Griem, H.R. 1974. Spectral line broadening by plasmas.New York: Acade mic Press, Inc.

Hoffman, J., Szy manski, Z., \& A zharonok, V. 2006. Plas ma Plume Induced During Laser Welding of Magnesium Alloys. . In: AIP Conference Proceedings., pp. 469-472 812,

Konjević, N. 1999, Plasma broadening and shifting of nonhydrogenic spectral lines: present status and applications, Physics Reports, 316 (6), 339-401. 
Milovanović, N., Dimitrijević, M.S., Popović, L.Č., \& Simić, Z. 2004. Importance of collisions with charged particles for stellar UV line shapes: Cd III. Astronomy and Astrophysics, 417, pp. 375-380.

Oertel, G.K., \& Shomo, L.P. 1968. Tables for the Calculation of Radial Multipole Matrix Elements by the Coulomb Approximation. Astrophysical Journal Supplement, 16, pp. 175-218.

Rauch, T., Ziegler, M., Werner, K., \& et al., 2007. Highresolution FUSE and HST ultraviolet spectroscopy of the white dwarf central star of Sh 2-216. Astronomy and Astrophysics, 1, pp. 317-329.

Rixon, G., Dubernet, M.L., Piskunov, N., \& et al., 2011. VAMDC: The Virtual Atomic and Molecular Data Centre: A New Way to Disseminate Atomic and Molecular DataVAMDC Level 1 Release. . In: AIP Conference Proceedings. , pp. 107-115 1344

Sahal-Bréchot, S. 1969. Impact theory of the broadening and shift of spectral lines due to electrons and ions in a plasma. Astronomy and Astrophysics, 1, pp. 91-123.

Sahal-Bréchot, S. 1969. Impact theory of the broadening and shift of spectral lines due to electrons and ions in a plasma. Astronomy and Astrophysics, 2, pp. 322-354. (continued).

Sahal-Bréchot, S. 1974. Stark broadening of isolated lines in the impact approximation. Astronomy and Astrophysics, 35, pp. 319-321.

Sahal-Bréchot, S. 1991. Broadening of ionic isolated lines by interactions with positively charged perturbers in the quasistatic limit. Astronomy and Astrophysics, 245, pp. 322330.

Sahal-Bréchot, S., Dimitrijević, M.S., \& Nessib, B.N. 2014. Widths and Shifts of Isolated Lines of Neutral and Ionized Atoms Perturbed by Collisions With Electrons and Ions: An Outline of the Semiclassical Perturbation (SCP) Method and of the Approximations Used for the Calculations. Atoms, 2, pp. 225-252.

Sahal-Bréchot, S., Dimitrijević, M.S., \& Moreau, N. 2017. STARK-B database.Observatory of Paris, LERMA / Astronomical Observatory of Belgrade. Retrieved from http://starkb.obspm.fr.

Sahal-Bréchot, S., Dimitrijević, M.S., Moreau, N., \& Nessib, B.N. 2015. The STARK-B database VAMDC node: A repository for spectral line broadening and shifts due to collisions with charged particles. Physica Scripta, 50, p. 8. 054008
Saloman, E.B. 2010. Energy Levels and Observed Spectral Lines of Ionized Argon, Ar II through Ar XVIII. Journal of Physical and Chemical Reference Data, 39(0331), p. 1.

Simić, Z., Dimitrijević, M.S., \& Kova cević, A. 2009. Stark broadening of spectral lines in chemically peculiar stars: Te I lines and recent calculations for trace elements. New Astronomy Review, 53(7-10), pp. 246-251.

Simić, Z., Dimitrijević, M.S., Milovanović, N., \& Sahal-Bréchot, S. 2005. a, Stark broadening of $\mathrm{Cd}$ I spectral lines. Astronomy and Astrophysics, 441(1), pp. 391-393.

Simic, Z., Dimitrijevic, M.S., Popovic, L.C., \& Dacic, M.D. 2005. Stark Broadening of F III Lines in Laboratory and Stellar Plasma. Journal of Applied Spectroscopy, 72(3), pp. 443-446. doi:10.1007/s 10812-005-0095-4

Simić, Z., Dimitrijević, M.S., Popović, L.Č., \& Dačić, M. 2006. Stark broadening parameters for Cu III, Zn III and Se III lines in laboratory and stellar plasma. New Astronomy, 12(3), pp. 187-191.

Sorge, S., Wierling, A., Röpke, G., Theobald, W., Sauerbrey, R., \& Wilhein, T. 2000. Diagnostics of a laser-induced dense plasma by hydrogen like carbon spectra. Journal of Physics B: Atomic, Molecular and Optical Physics, 33(16), pp. $2983-$ 3000. doi:10.1088/0953-4075/33/16/304

Tankosić, D., Popović, L.Č., \& Dimitrijević, M.S. 2003. The electron-impact broadening parameters for Co III spectral lines. Astronomy and Astrophysics, 399, pp. 795-797.

Taresch, G., Kudritzki, R.P., Hurwitz, M., \& et al., 1997. Quantitative analysis of the FUV, UV and optical spectrum of the O3 star HD 93129A. Astronomy and Astrophysics, 321, pp. 531-548.

Torres, J., van de Sande, M.J., van der Mullen, J.J.A.M., Gamero, A., \& Sola, A. 2006. Stark broadening for simultaneous diagnostics of the electron density and temperature in atmospheric microwave discharges. Spectrochimica Acta, Part B: Atomic Spectroscopy, 61(1), pp. 58-68.

van Regemorter, H., Binh, H.D., \& Prud'homme, M. 1979. Radial transition integrals involving low or high effective quantum numbers in the Coulomb approximation. Journal of Physics B: Atomic, Molecular and Optical Physics, 12, pp. 1053-1061.

Werner, K., Rauch, T., \& Kruk, J.W. 2007. Discovery of photospheric argon in very hot central stars of planetary nebulae and white dwarfs. Astronomy and Astrophysics, 466, pp. 317-322.

Griem, H.R. 1992. Plas ma spectroscopy in inertial confinement fusion and soft x-ray laser research. Physics of Fluids, 4(7), str. 2346-2361. 